\title{
In situ methodology for studying state driver stress: A between-subjects design replication
}

\author{
Wickens, C. M., Wiesenthal, D. L., \& Roseborough, J. E. W.
}

Version Post-Print/Accepted Manuscript

Citation Wickens, C. M., Wiesenthal, D. L., \& Roseborough, J. E. W. (2015). In

(published version) situ methodology for studying state driver stress: A between-subjects design replication. Journal of Applied Biobehavioral Research, 20, 3751.

Publisher's Statement This is the peer reviewed version of the following article: Wickens, C. M., Wiesenthal, D. L., \& Roseborough, J. E. W. (2015). In situ methodology for studying state driver stress: A between-subjects design replication. Journal of Applied Biobehavioral Research, 20, 37-51, which has been published in final form at https://dx.doi.org/10.1111/jabr.12029. This article may be used for non-commercial purposes in accordance with Wiley Terms and Conditions for Self-Archiving.

\section{How to cite TSpace items}

Always cite the published version, so the author(s) will receive recognition through services that track citation counts, e.g. Scopus. If you need to cite the page number of the TSpace version (original manuscript or accepted manuscript) because you cannot access the published version, then cite the TSpace version in addition to the published version using the permanent URI (handle) found on the record page. 


\author{
In Situ Methodology for Studying State Driver Stress: \\ A Between-Subjects Design Replication \\ Christine M. Wickens, \\ Centre for Addiction and Mental Health \\ David L. Wiesenthal and James E. W. Roseborough \\ York University
}

\begin{abstract}
Author Note
Christine M. Wickens, Social and Epidemiological Research Department, Centre for Addiction and Mental Health; David L. Wiesenthal, Department of Psychology, York University; James E. W. Roseborough, Department of Psychology, York University.

Christine M. Wickens was supported by a student scholarship from the Canadian Transportation Research Forum and by postdoctoral fellowships from the Social Sciences and Humanities Research Council and the Centre for Addiction and Mental Health. David L. Wiesenthal held a Social Sciences and Humanities Research Council grant which facilitated this research. The authors would like to thank Vadim Paskarou, Arathi Packiyanathan, Meenu Minhas, Lisa Hilliard, Siamak Riazzi, and Sara Howard for their assistance with participant recruitment and data entry.

Correspondence concerning this article should be addressed to Christine Wickens, Social and Epidemiological Research Department, Centre for Addiction and Mental Health, 33 Russell Street, Toronto, Ontario M5S 2S1 Canada Email: Christine.Wickens@camh.ca
\end{abstract}




\begin{abstract}
Previous studies of driver stress have utilized in-vehicle in situ questionnaires to measure driver stress during the actual commute. These studies demonstrated several important findings, but all adopted a repeated-measures research design where each participant was exposed to counterbalanced high and low congestion conditions. This approach reduced between-subjects variability but increased the possibility of demand characteristics. The current study replicated the findings of the in situ methodology using a between-subjects research design. State stress was greater in heavy traffic. Time urgency, lack of perceived control, and trait susceptibility to perceiving driving as stressful contributed to higher levels of state driver stress. No gender differences in state driver stress were found. Implications of the results and future research directions are discussed.
\end{abstract}

Keywords: Driver stress, aggression, traffic congestion, control, gender 
In Situ Methodology for Studying State Driver Stress:

A Between-Subjects Design Replication

\section{Driver Stress}

Driver stress occurs when a motorist appraises a driving situation as demanding or dangerous (Gulian, Matthews, Glendon, Davies, \& Debney, 1989; Matthews, Dorn, \& Glendon, 1991). The stress generally results in emotional responses including anxiety and frustration (Gulian, Debney, Glendon, Davies, \& Matthews, 1989; Gulian, Matthews, et al., 1989), as well as psychophysiological responses such as increased blood pressure and muscle tension (Malta et al., 2001; White \& Rotton, 1998). Motorists who describe driving as highly stressful demonstrate reduced control skills and increased errors when following another vehicle (Matthews et al., 1998) and are involved in more minor traffic crashes (Matthews et al., 1991; Selzer \& Vinokur, 1975). Driver stress is also associated with an increased risk of driver aggression including tailgating, swearing/yelling, and obscene hand gestures (Hennessy \& Wiesenthal, 2001a). In a study that asked participants to describe and rate roadway events experienced during their daily commute, up to $75 \%$ of participant responses to driver stress involved aggressive driver behaviour (Gulian, Debney, et al., 1989). Likewise, in a survey where $85 \%$ of respondents acknowledged engaging in aggressive roadway behavior in the past year, $72 \%$ cited stress and frustration as the cause of their behavior (Drivers.com, 2007).

\section{Risk Factors for Driver Stress}

Much scientific literature has focused on the identification of risk factors or variables that contribute to driver stress including characteristics of the driver (e.g., demographics, personality traits, mental health), features of the environment or situation 
(e.g., weather, vehicle type), and interactions between these person-related and environmental variables (Wickens, Mann, \& Wiesenthal, 2013). Many of these studies utilize retrospective questionnaires that ask motorists to reflect on their previous roadway experiences. These studies provide valuable insights, but they also face a serious potential limitation: biased or inaccurate recall. Drivers may not accurately recall all of the sources of roadway frustration nor the intensity of stress or anger experienced.

In response to this limitation, Hennessy, Wiesenthal and colleagues initiated a program of research that utilized an in-vehicle in situ methodology (e.g., Hennessy \& Wiesenthal, 1997, 1999, 2001b; Hennessy, Wiesenthal, \& Kohn, 2000; Wickens \& Wiesenthal, 2005; Wiesenthal, Hennessy, \& Totten, 2000). In the earliest studies, highway/expressway commuters identified a portion of their route where they typically encountered light or easygoing traffic and a portion of their route where they typically encountered heavy traffic congestion. The order in which participants encountered these levels of traffic congestion was counterbalanced. While participants were driving through these portions of their route on their way home, they would use a cellular telephone to call the experimenter. Driver stress would be measured via telephone during the actual commute. In the latter studies, ethical considerations concerning the safety of cellular telephone use while driving led to a minor amendment to the procedure, such that commuters were asked to exit the highway after driving through the predetermined traffic zone, safely park their vehicle, and telephone the experimenter. In either case, this methodology minimized the risk of biased or inaccurate recall.

The studies utilizing this methodology replicated several important findings. First, traffic congestion is a major source of commuter stress. Participants perceived greater 
stress when driving through heavy traffic congestion compared to light traffic. Second, time urgency predicted driver stress; the more participants were rushed for time, the more driver stress they reported. Third, perceived control has been recognized as improving well-being in a number of contexts including healthcare, workplace, and academic settings (Skinner, 1996). Perceived control has a similar function in the driving environment; the more participants felt they were in control of the driving situation, the less driver stress they reported. Fourth, no gender effects were reported for driver stress or mild driver aggression. Male participants reported greater roadway violence than female participants, but only in association with a strongly vengeful attitude (Hennessy \& Wiesenthal, 2002a, 2002b). Finally, participants who had a higher baseline level of stress reported more driver stress in the actual driving situation. Gulian, Debney, et al. (1989) conceptualized driver stress as both a state-level construct, specific to a given situation, and as a trait-level construct, which reflected an accumulation of negative feelings and frustrations related to driving generally. Gulian, Debney, et al. postulated a reciprocal relationship between the state- and trait-level constructs, with each exacerbating and reinforcing the other. Hennessy, Wiesenthal, and colleagues confirmed the positive association between these state- and trait-level constructs within the actual driving environment.

\section{Limitations of Previous Research}

Despite these important findings and the identification of additional risk factors for driver stress (e.g., daily hassles, job stress, general aggression), the studies by Hennessy, Wiesenthal, and colleagues shared a common limitation. Each of these studies adopted a repeated-measures research design, requiring that each participant be exposed 
to both high and low congestion conditions. This approach was taken in order to reduce between-subjects variability, which could have been overwhelming in a study conducted outside of the strict experimental control of the laboratory. However, the repeatedmeasures design also increased the possibility of demand characteristics, as participants could have deliberately inflated their driver stress scores in the more congested traffic area or deflated scores in the less congested traffic area. Although the original studies all tested for order effects and found none, participants were aware in advance that they would be subjected to contrasting congestion levels and may have speculated about the experimenters' expected findings.

Purpose and Hypotheses of the Current Study

In response to this criticism, the current study is a replication of the primary findings of the previous research using a between-subjects research design. As such, the follow hypotheses were postulated:

1. Time urgency would be associated with increased state driver stress.

2. Perceived control would be associated with reduced state driver stress.

3. Increased traffic congestion would be associated with increased state driver stress.

4. Trait driver stress would positively predict state driver stress.

5. Gender would not be predictive of state driver stress.

Method

\section{Participants}

A total of 170 undergraduate students were recruited to participate in a study of thoughts and feelings about driving. Participants were required to (1) drive home alone; 
(2) along a 400- or similar series highway; and (3) to encounter a section of exclusively heavy or exclusively light traffic on that highway. In Ontario, the 400-series highways are a controlled-access network of multi-lane highways with a speed limit of $100 \mathrm{kph}$ (62 mph). Participants were obtained using the undergraduate research participant pool and through on-campus in-person recruitment. Respondents recruited through the participant pool received course credit; respondents recruited in-person received a $\$ 10$ university bookstore gift card or a certificate for a free cinema movie.

As with previous studies using the in situ methodology, the level of traffic congestion encountered by participants was estimated in advance of their participation based on their recall of typical daily commutes. However, estimates of the level of traffic are not always accurate; drivers" perceptions of what constitutes "heavy traffic" can vary considerably (Laetz, 1990). Also, the level of traffic encountered during participation sometimes differs from the expected norm (e.g., a collision results in heavy traffic where there is normally only light traffic). Given the median reported speed of $70 \mathrm{kph}$ across both conditions (43.5 $\mathrm{mph}$ ), participants in the heavy congestion condition were required to travel no faster than $69 \mathrm{kph}$ and participants in the light traffic condition were required to travel no slower than $70 \mathrm{kph}$. Participants who did not meet this requirement on the day of their participation were eliminated from the analysis. A total of 170 participants completed the study; based on this inclusion criteria, the current analysis includes a sample of 134 participants.

\section{Measures}

Trait Driver Stress. The Driving Behavior Inventory-General (DBI-Gen; Gulian, Matthews, et al., 1989) was used to measure participants' general disposition or trait 
susceptibility to driver stress. The current study used an 11-item version of the DBI-Gen, which has been previously established as a valid, robust, and reliable measure (Glendon et al., 1993; Hennessy \& Wiesenthal, 1997; Matthews et al., 1991). The 11-item version of the measure, which uses a rating scale ranging from 0 to 100 , has demonstrated strong internal consistency in prior research $(\alpha=.80-.90$; Hennessy \& Wiesenthal, 1997; Hennessy et al., 2000).

State Driver Stress. The State Driver Stress Questionnaire (SDSQ; Hennessy \& Wiesenthal, 1997) was designed to measure the state-level experience of driver stress through verbal administration in the actual driving situation. The scale consists of 11 items that are similar to those from the DBI-Gen, as well as 10 items from the Stress Arousal Checklist (Mackay, Cox, Burrows, \& Lazzerini, 1978). The SDSQ, which uses a rating scale ranging from 0 to 100 , has demonstrated strong internal consistency in both light $(\alpha=.92-.94)$ and heavy $(\alpha=.90-.97)$ traffic congestion conditions (Hennessy \& Wiesenthal, 1997, 1999; Hennessy et al., 2000; Wiesenthal et al., 2000).

Time Urgency. A 3-item measure of time urgency used in previous research (Hennessy \& Wiesenthal, 1997; Wickens \& Wiesenthal, 2005) was included in the current study. Level of agreement with each of the items was rated on a scale ranging from 0 to 100.

Perceived Control. A 4-item measure of perceived control over the driving environment was also included in the current study. This measure was revised from previous research (e.g., Wickens \& Wiesenthal, 2005) in which this scale consisted of only a single item. Again, level of agreement with each item was rated on a scale ranging from 0 to 100. 
Level of Traffic Congestion Check. In order to confirm that participants correctly predicted the level of traffic congestion encountered during their commute, one item asked participants to report their average speed in the last 5 minutes of their commute.

Demographic and Additional Information. Demographic information obtained included age, gender, marital status, number of years licensed to drive, and number of hours spent driving each week. A series of other individual difference measures were also included for piloting purposes. As they are not relevant to the current analysis, these measures will not be addressed here.

\section{Procedure}

The procedures followed in the current study were very similar to those of the Hennessy and Wiesenthal research previously cited. During recruitment, participants identified an area along their highway commute where they typically encountered either light traffic or heavy congestion. The participants' experience of light or heavy traffic would need to be expected to persist for at least five continuous minutes in order to make them eligible for participation. Recognizing that perceptions of heavy traffic congestion can vary (Laetz, 1990), and that participants self-identifying themselves as motorists who experience heavy traffic may have encountered what was defined as light congestion for the purposes of the current study (i.e., traffic flowing at a speed of $70 \mathrm{kph}$ or more), it was anticipated that more participants from the heavy traffic congestion condition would be eliminated from the current analysis than participants from the light traffic condition. As well, more potential participants experienced light than heavy traffic congestion during their commute. For these reasons, participants who qualified for both the light and heavy traffic congestion conditions were assigned to the heavy traffic group. Participants 
were asked to identify a highway exit near the end of the light or heavy traffic area and a parking spot where they could safely complete a short questionnaire. Selection of this location in advance of their participation prevented any concern about parking from contributing to their state driver stress.

Participants were given two packages of questionnaires. The first package, labeled "Before You Leave School”, contained the DBI-Gen and demographic questionnaire. Participants were instructed to complete this questionnaire prior to their afternoon commute between campus and home on the day of their study participation. The second package, labeled "Driving Session", contained the SDSQ. Unlike previous studies in this line of research which relied on cell phone administered measures, the current study administered the SDSQ using a paper-and-pencil survey method. Participants were instructed to drive through their identified traffic area as they normally would, to leave the highway at the designated exit, and to proceed to their preplanned parking spot. Once safely stopped, participants responded to the SDSQ. Upon completion of this questionnaire, participation in the study ended. Participants were asked to return the questionnaire packages to the experimenter on their next visit to campus. This methodology was approved by the York University Research Ethics Board.

Results

\section{Demographics}

Of the 63 participants recruited for the light traffic condition, 58 participants encountered traffic moving at a speed of at least $70 \mathrm{kph}$ on the day of their participation and were retained for the current analysis. Of the 106 participants recruited for the heavy congestion condition, 76 participants encountered traffic moving slower than $70 \mathrm{kph}$ on 
the day of their participation and were retained for the current analysis. One participant was excluded from the analysis because his traffic condition could not be determined. As expected, more participants from the heavy traffic congestion condition were eliminated from the current analysis than participants from the light traffic condition, $\chi^{2}(1)=9.98, p$ $=.002$, Cramer's $\mathrm{V}=.24$. In terms of other predictor variables and demographic characteristics, a series of independent samples $t$-tests and Pearson chi-square analyses contrasting the included versus eliminated participants revealed no significant differences between groups.

The final sample consisted of 55 men and 78 women (one participant did not respond). Of the 55 male participants, 24 (44\%) were in the light traffic congestion condition. Of the 78 female participants, 33 (42\%) were in the light traffic congestion condition. Their ages ranged from 17 to 44 years, with a mean age of 23.4 years $(S D=$ 5.67). Participants had been licensed to drive for a mean of 6.2 years $(S D=4.68)$ and reported driving a mean of 1.7 hours per day $(S D=0.99)$. With the exception of average hours driven per day, demographic characteristics did not significantly differ across the light and heavy traffic congestion groups. Participants in the heavy traffic condition reported driving an average of 1.9 hours per day $(S D=1.05)$, whereas participants in the light traffic condition reported driving an average of 1.6 hours per day $(S D=0.87), t(127)$ $=-2.00, p=.048$.

\section{Reliability of the Measures}

The internal consistency of all continuous measures (see Table 1) including the SDSQ, time urgency, perceived control, and the DBI-Gen was within the acceptable 
range, each generating a Cronbach's alpha greater than the recommended minimum of .70 (Nunnally \& Bernstein, 1994).

\section{Bivariate Relationships among Variables}

Table 1 presents an intercorrelational matrix of the bivariate relationships between continuous variables. A positive relationship was found between state driver stress and time urgency $(r=.50, p<.001)$, whereas a negative relationship was found between state driver stress and perceived control $(r=-.54, p<.001)$. In light of these relationships, both time urgency and perceived control were treated as covariates in the subsequent hierarchical linear regression analysis.

A series of independent samples $t$-tests assessing gender differences in the continuous variables found no significant results. As a lack of a gender difference in state driver stress was specifically hypothesized, a test of equivalence was used to assess this result. The mean SDSQ score for male participants was $40.65(S D=18.11)$ and the mean SDSQ score for female participants was $42.54(S D=21.02)$. The two groups were statistically equivalent using the Schuirmann two independent samples test of equivalence (Schuirmann, 1987) with an interval of one-half of a standard deviation, $t 1$ $(125.74)=-3.46, p<.001, t 2(125.74)=2.35, p=.01$, epsilon $=9.91$, Cohen's $d=.10$. Thus, the null hypothesis that the difference between the means exceeds the equivalence interval was rejected.

A $t$-test assessing trait driver stress as a function of congestion level also revealed no significant difference between groups, $t(132)=-.86, p=.39$. A Pearson chi-square test found no significant difference in the number of male and female drivers across the light and heavy traffic conditions, $\chi^{2}(1)=.02, p=.88$. 


\section{Hierarchical Linear Regression Analysis}

The main effect predictors assessed in the regression analysis of state driver stress included congestion level, gender, and trait driver stress. Preliminary screening of the data revealed that perceived control (a covariate) was negatively skewed. In response to this assumption violation, perceived control scores were reflected and a square root transformation applied, thereby resolving non-normality. As a result of this transformation, higher perceived control scores within the regression now represent an absence of perceived control. This change is reflected in the relabeling of the variable as "Lack of Perceived Control". The three-way interaction among the main predictor variables and all two-way interactions were included in each initial model. In order to reduce multicollinearity, all interactions were centered. Main effects were also centered, although Kline and Dunn (2000) found identical results with centered and non-centered main effects when conducting regression analysis.

A hierarchical stepwise regression analysis was used to generate final models that included only two classes of effects: those that were statistically significant and those that, significant or not in themselves, were implicated in a significant interaction. This statistical approach has been outlined in greater detail elsewhere (e.g., Kohn, Gurevich, Pickering, \& Macdonald, 1994; Kohn \& Macdonald, 1992) and was adopted in the original in situ methodology studies using the repeated-measures design conducted by Hennessy, Wiesenthal, and colleagues (e.g., Hennessy \& Wiesenthal, 1997, 1999, 2001b; Hennessy et al., 2000; Wickens \& Wiesenthal, 2005; Wiesenthal et al., 2000). This strategy was originally chosen because of its greater statistical power to delete noncontributory predictor variables. Using this approach, all main effects and two-way 
interactions are entered forcibly and the three-way interaction is entered stepwise on the first run. If the three-way interaction is significant, all significant and implicated variables are retained. If the three-way interaction is not significant, all main effects are entered forcibly and the two-way interactions are entered stepwise on the second run. This pattern is followed until, if necessary, all main effects are entered stepwise on the third run.

Table 2 presents the final regression model. Missing cases were treated listwise, resulting in a loss of two additional participants. As predicted, time urgency and a lack of perceived control positively predicted state driver stress. Likewise, trait driver stress contributed to a greater experience of state driver stress. Finally, heavy traffic was associated with higher levels of state driver stress. Overall, the model accounted for $64 \%$ of the variance. Although it was not statistically significant and was therefore eliminated from the final model, the interaction between congestion level and trait driver stress approached significance $(p=.07)$ in the second run of the regression. Increased state driver stress, especially in heavy traffic, was associated with trait driver stress.

\section{Alternative Traffic Congestion Criteria Tested}

The median reported speed of $70 \mathrm{kph}$ was chosen as the speed criterion for inclusion in the statistical analysis; participants in the heavy congestion condition were required to be traveling less than $70 \mathrm{kph}$ and participants in the light traffic condition were required to be traveling at least $70 \mathrm{kph}$ immediately prior to completing the invehicle questionnaire. This speed criterion was founded on both a theoretical and practical basis. In an earlier study using the in situ methodology, Wickens and Wiesenthal (2005) found that participants reported travelling $63.83 \mathrm{kph}$ on average in their heavy congestion condition, suggesting that a criterion of $70 \mathrm{kph}$ in the current 
study would be appropriate. In addition, the value of $70 \mathrm{kph}$ maximized the number of participants retained for the current analysis. Nonetheless, additional analyses were conducted to confirm the findings of the current study using two alternative traffic congestion criteria; 60 and $80 \mathrm{kph}$. Specifically, the regression analyses were re-run twice, requiring participants in the heavy congestion condition to report traveling no more than 59 and $79 \mathrm{kph}$, and participants in the light traffic condition to report traveling no less than 60 and $80 \mathrm{kph}$. The regression analyses using these alternative criteria produced similar results to those reported previously; however, the interaction between congestion level and trait driver stress that approached significance in the second run of the original regression was no longer marginally significant $(p=.14)$ when the $60 \mathrm{kph}$ criterion was introduced.

\section{Discussion}

The in-vehicle in situ methodology for quasi-experimental study of state driver stress developed by Hennessy, Wiesenthal, and colleagues (e.g., Hennessy \& Wiesenthal, 1997, 1999, 2001b; Hennessy et al., 2000; Wickens \& Wiesenthal, 2005; Wiesenthal et al., 2000) has contributed substantially to the available scientific literature concerning driver stress and aggression. Despite this significant contribution, the methodology was not fully demonstrated as it had been used exclusively with a repeated-measures research design. As a result, the methodology was criticized for possibly increasing demand characteristics; drivers may have inflated their stress scores in the more congested traffic area or deflated their stress scores in the less congested traffic area. In response to this limitation, the current study conducted a replication of the previous research of state driver stress using the in situ methodology and a between-subjects research design. 
Results of the current study replicated those of the previous research, demonstrating the strength and utility of the in situ methodology regardless of the research design. Consistent with the first hypothesis, drivers experiencing time urgency reported higher levels of state driver stress. Lazarus (1981) postulated an additive effect of one stressful event on the perceived severity of another. Everyday hassles such as getting to work/school on time likely heighten the frustration and negative affect in the driving environment. Likewise, consistent with the second hypothesis, drivers experiencing a lack of perceived control over the driving situation also reported higher levels of state driver stress. Perceived control over one's environment is an important factor in the stress appraisal process (Glass \& Singer, 1972), and it has been suggested that perceiving adverse driving conditions as stressful is the immediate result of a loss of control (Gulian, Matthews, et al., 1989). Consistent with the third hypothesis, heavy traffic congestion was associated with reports of greater state driver stress. Traffic congestion blocks the attainment of goals, such as driving at a certain speed, and is therefore interpreted as a negative and frustrating event (Novaco, Stokols, Campbell, \& Stokols, 1979). Even without time demands, a significant percentage of drivers frequently report irritation in congested roadway conditions (Gulian, Debney, et al., 1989). Consistent with the fourth hypothesis, drivers with a trait susceptibility to driver stress reported higher levels of in-vehicle state stress. High trait-stress drivers are more likely to interpret negative roadway events as stressful and to show heightened levels of arousal and unpleasant mood when driving (Gulian, Matthews, et al., 1989; Matthews et al., 1998), which, in turn, maintains and/or intensifies in a cyclical fashion their general disposition to driver stress (Hennessy et al., 2000). Finally, consistent with previous 
studies in this research program (Hennessy \& Wiesenthal, 1997, 1999; Hennessy et al., 2000; Wickens \& Wiesenthal, 2005; Wiesenthal et al., 2000), male and female participants reported similar levels of state driver stress. Although male drivers may commit more driving violations, receive more traffic citations, and be involved in more motor vehicle collisions than female drivers, when not taking exposure into account, there is evidence to suggest that male and female drivers experience similar overall levels of stress and anger in response to roadway frustrations. The difference lies in the nature and severity of their behavioral response to these emotions (Wickens et al., 2012).

Interestingly, the interaction between congestion and trait driver stress approached significance in the second round of the regression, but ultimately, was deleted from the final model. Hennessy and Wiesenthal (1997) conducted a median split of participants' DBI-Gen scores and entered them into a split-plot factorial analysis with level of traffic congestion as the within-subjects variable and trait driver stress as the between-subjects variable. Similar to the marginally significant interaction in the current analysis, Hennessy and Wiesenthal identified a significant interaction between trait driver stress and congestion level, such that high trait stress drivers experienced more state driver stress, particularly under heavy traffic congestion conditions. According to Hennessy and Wiesenthal, this interaction had implications for the distinction between state- and traitlevel measures of driver stress. Specifically, the DBI-Gen proved to be a good indicator of which drivers do and do not have a general disposition to driver stress. The fact that a more detailed picture of driver stress emerged when the level of traffic within the driving environment was considered suggests that researchers must be careful in using the DBIGen appropriately. Researchers may consider providing contextual information 
concerning specific roadway conditions (e.g., level of traffic, time urgency) if they intend to examine trait-level stress at any level of further situation specificity. As the interaction in the current study only approached significance, future research is needed to determine if this effect is an artifact or a function of the research design.

Despite addressing concerns of an increased risk of demand characteristics associated with the repeated-measures design, the current study was still affected by several shortcomings. First, although the potential for demand characteristics was reduced by the between-subjects design, it may not have been eliminated completely. Participants were assigned to conditions after providing information about their commute, and were aware of both study groups. Although participants were not able to adjust responses in one condition relative to another, participants could have adjusted their responses based on knowledge of group assignment alone. Future research should find a way to obtain participants' commuting details without revealing information about treatment groups to participants. Prescreening in a large participant pool may help to address this issue. Second, as with previous studies using this in situ methodology, there was no confirmation that participants had actually parked their vehicles mid-commute and completed the in-vehicle questionnaire as instructed. The recent accessibility of Global Positioning System (GPS) technology has now made participant tracking possible. Assuming potential ethical concerns could be addressed, GPS technology could be used in future studies to confirm reported traffic levels and participant compliance. Third, as with the previous studies in this research program, driver stress was measured during a single trip. The way in which personality, situation, and environment interact to affect driver stress may be more complex than can be measured in a single journey. Research 
with multiple measurements is needed to understand in greater detail the true nature of the relationship between these constructs. Fourth, the current sample consisted primarily of younger drivers. Previous research has typically shown younger drivers to report greater stress and aggression in the roadway environment (Gulian, Debney, et al., 1989; Wickens, Mann, Stoduto, Ialomiteanu, \& Smart, 2011). Future research that includes a wider array of driver ages is needed to ensure that the findings of the current study are generalizable to the entire driving population. Finally, the selection of participants to be retained for the current analysis was based on a median split of self-reported speed driven just prior to completing the in-vehicle questionnaire. A tertiary split would have ensured a greater qualitative difference between the experiences of drivers in the light versus heavy traffic conditions. Although the median split allowed for maximal retention of participants, future research might consider recruiting a larger sample and adopting a more tertiary speed requirement (e.g., no slower than $80 \mathrm{kph}$ for the light traffic condition, no faster than $30 \mathrm{kph}$ for the heavy traffic condition) in order to maximize the study's ability to detect small effects. For instance, the interaction between congestion level and trait driver stress may be better detected with more stringent speed criteria. Results of the current study have several important implications for the societal response to driver stress, not the least of which is acknowledging the contribution that traffic congestion and time urgency make to driver stress, and recognizing them as a public health concern. As such, a multifaceted approach to reducing driver stress should be implemented including organizational changes to reduce traffic congestion (e.g., telecommuting, flextime hours) and promotional campaigns encouraging motorists to remain calm behind the wheel (e.g., deep breathing, music listening (Wiesenthal et al., 
2000), etc.). Based on the results of the current study, a campaign focused on reducing driver stress would not need to target male and female motorists separately. Instead, such a campaign could target drivers who have developed a trait-level disposition of driver stress to recognize and address their susceptibility to negative affect behind the wheel. These efforts to reduce driver stress could go a long way to reducing the incidence of risky driving, roadway aggression, and motor vehicle collisions. 


\section{References}

Drivers.com (2007, June 26). Canadian drivers' multi-tasking fuels aggression: What are Canadians really doing behind the wheel? Retrieved 2010, August 23, from http://www.drivers.com/article/429/.

Glass, D. C., \& Singer, J. E. (1972). Urban stress. NewYork: Academic Press.

Glendon, A. I., Dorn, L., Matthews, G., Gulian, E., Davies, D. R., \& Debney, L. M. (1993). Reliability of the Driving Behaviour Inventory. Ergonomics, 36, 719-726.

Gulian, E., Debney, L. M., Glendon, A. I., Davies, D. R., \& Matthews, G. (1989). Coping with driver stress. In F. J. McGuigan, W. E. Sime, \& J. M. Wallace (Eds.), Stress and tension control 3: Stress management (pp. 173-186). New York: Plenum Press.

Gulian, E., Matthews, G., Glendon, A. I., Davies, D. R., \& Debney, L. M. (1989). Dimensions of driver stress. Ergonomics, 32, 585-602.

Hennessy, D. A., \& Wiesenthal, D. L. (1997). The relationship between traffic congestion, driver stress and direct versus indirect coping behaviours. Ergonomics, 40, 348-361. doi: 10.1080/001401397188198

Hennessy, D. A., \& Wiesenthal, D. L. (1999). Traffic congestion, driver stress, and driver aggression. Aggressive Behavior, 25, 409-423.

Hennessy, D. A., \& Wiesenthal, D. L. (2001a). Gender, driver aggression, and driver violence: An applied evaluation. Sex Roles, 44, 661-676.

Hennessy, D. A., \& Wiesenthal, D. L. (2001b). Further validation of the Driving Vengeance Questionnaire. Violence \& Victims, 16, 565-573. 
Hennessy, D. A., \& Wiesenthal, D. L. (2002a). Aggression, violence, and vengeance among male and female drivers. Transportation Quarterly, 56, 65-75.

Hennessy, D. A., \& Wiesenthal, D. L. (2002b). The relationship between driver aggression, violence, and vengeance. Violence and Victims, 17, 707-718.

Hennessy, D. A., Wiesenthal, D. L., \& Kohn, P. M. (2000). The influence of traffic congestion, daily hassles, and trait stress susceptibility on state driver stress: An interactive perspective. Journal of Applied Biobehavioral Research, 5, 162-179.

Kline, T. J. B., \& Dunn, B. (2000). Analysis of interaction terms in structural equation models: A non-technical demonstration using the deviation score approach. Canadian Journal of Behavioural Science, 32, 127-132.

Kohn, P. M., Gurevich, M., Pickering, D. I., \& Macdonald, J. E. (1994). Alexithymia, reactivity, and the adverse impact of hassles based stress. Personality and Individual Differences, 16, 805-812.

Kohn, P. M., \& Macdonald, J. E. (1992). Hassles, anxiety, and negative wellbeing. Anxiety, Stress, and Coping, 5, 151-163.

Laetz, T. J. (1990). Predictions and perceptions: Defining the traffic congestion problem. Technological Forecasting and Social Change, 38, 287-292.

Lazarus, R. S. (1981). Little hassles can be hazardous to health. Psychology Today, July, $58-62$.

Mackay, C. J., Cox, T., Burrows, C. G., \& Lazzerini, A. J. (1978). An inventory for the measurement of self-reported stress and arousal. British Journal of Social and Clinical Psychology, 17, 283-284. 
Malta, L. S., Blanchard, E. B., Freidenberg, B. M., Galovski, T. E., Karl, A., \& Holzapfel, S. R. (2001). Psychophysiological reactivity of aggressive drivers: An exploratory study. Applied Psychophysiology and Biofeedback, 26, 95-116.

Matthews, G., Dorn, L., \& Glendon, A. I. (1991). Personality correlates of driver stress. Personality and Individual Differences, 12, 535-549.

Matthews, G., Dorn, L., Hoyes, W., Davies, D. R., Glendon, A. I., \& Taylor, R. G. (1998). Driver stress and performance on a driving simulator. Human Factors, 40, $136-149$.

Novaco, R. W., Stokols, D., Campbell, J., \& Stokols, J. (1979). Transportation, stress, and community psychology. American Journal of Community Psychology, 7, $361-380$.

Nunnally, J. C., \& Bernstein, I. H. (1994). Psychometric theory ( $3^{\text {rd }}$ ed.). Toronto: McGraw-Hill, Inc.

Schuirmann, D. J. (1987). A comparison of the two one-sided tests procedure and the power approach for assessing equivalence of average bioavailability. Journal of Pharmacokinetics and Biopharmaceutics, 15, 657-680.

Selzer, M. L., \& Vinokur, A. (1975). Role of life events in accident causation. Mental Health and Society, 2, 36-54.

Skinner, E. A. (1996). A guide to constructs of control. Journal of Personality and Social Psychology, 71, 549-570.

White, S. M., \& Rotton, J. (1998). Type of commute, behavioral aftereffects, and cardiovascular activity: A field experiment. Environment and Behavior, 30, 763780. 
Wickens, C. M., Mann, R. E., Stoduto, G., Butters, J. E., Ialomiteanu, A., \& Smart, R. G. (2012). Does gender moderate the relationship between driver aggression and its risk factors? Accident Analysis and Prevention, 45, 10-18.

Wickens, C. M., Mann, R. E., Stoduto, G., Ialomiteanu, A., \& Smart, R. G. (2011). Age group differences in self-reported road rage perpetration and victimization. Transportation Research Part F: Traffic Psychology and Behaviour, 14, 400-412.

Wickens, C. M., Mann, R. E., \& Wiesenthal, D. L. (2013). Addressing driver aggression: Contributions from psychological science. Current Directions in Psychological Science, 22, 386-391.

Wickens, C. M., \& Wiesenthal, D. L. (2005). State driver stress as a function of occupational stress, traffic congestion, and trait stress susceptibility. Journal of Applied Biobehavioral Research, 10, 83-97.

Wiesenthal, D. L., Hennessy, D. A., \& Totten, B. (2000). The influence of music on driver stress. Journal of Applied Social Psychology, 30, 1709-1719. 
Table 1

Intercorrelations, Means, and Standard Deviations of Continuous Variables

\begin{tabular}{lcccc}
\hline Variables & 1 & 2 & 3 & 4 \\
\hline 1. State Driver Stress Questionnaire (SDSQ) & - & & \\
2. Time urgency & $.50^{* * *}$ & - & \\
3. Perceived control & $-.54 * * *$ & -.14 & - & \\
4. Trait driver stress (DBI-Gen) & $.52 * * *$ & $.20 *$ & -.09 & - \\
Mean & 41.64 & 45.78 & 64.03 & 49.41 \\
$S D$ & 19.79 & 24.67 & 23.42 & 18.95 \\
Number of items & 21 & 3 & 4 & 11 \\
$\alpha$ & .94 & .72 & .75 & .86 \\
\hline$* p<.05, * * * p<.001$. & & & &
\end{tabular}


Table 2

Final Regression Model Predicting State Driver Stress (SDSQ; $n=132$ )

\begin{tabular}{lcccccc}
\hline & \multicolumn{3}{c}{ Unstandardized } & Standardized & & \\
\cline { 2 - 4 } Variables & $B$ & $S E B$ & $\beta$ & & \multirow{2}{*}{$*$} & $R^{2}$ Change \\
\cline { 2 - 5 } Time urgency & 0.27 & 0.04 & .35 & 6.39 & \\
Lack of perceived control & 3.81 & 0.55 & .40 & 6.94 & $.497^{* * *}$ \\
Trait driver stress (DBI-Gen) & 0.39 & 0.06 & .38 & & 7.07 & $.135^{* * *}$ \\
Congestion level & 6.60 & 2.28 & .17 & & 2.90 & $.023^{* *}$ \\
\hline
\end{tabular}

Note: $F(4,127)=60.05, p<.001$, adjusted $R^{2}=.643$.

$* * p<.01, * * * p<.001$. 